\title{
Low Cost, Cross-language and Cross-platform Information Retrieval and Documentation Tools
}

\author{
Nikitas N. Karanikolas \\ Department of Informatics, Technological Educational Institute of Athens, Greece
}

In this paper we focus on the design and implementation of low cost, cross language and cross platform information retrieval and documentation tools suitable for the collection, organization and administration of unstructured and semi-structured information imported from various sources. A modular Computer-Assisted Information Resources Navigation (CAIRN) software architecture is proposed and the requirements of each module are presented. A discussion about the implementation is based on the experiments made with a prototype of such a software tool. The technologies that are incorporated into the modern operating systems and the opportunities that they offer for implementing the modules of the CAIRN architecture are also examined and evaluated. Some of these technologies are common / independent from the operating systems, while some others are distinctive. In this latter case we face barriers (restrictions) to a straightforward implementation of the CAIRN software systems in the whole range of desktop operating systems (e.g. Windows, Mac OS, Linux, Solaris). Some alternative technologies are presented to avoid this serious constraint. Evaluation of the implementation effort is also discussed and eventually some conclusions and future plans for further improvement of the CAIRN architecture are given.

Keywords: free text retrieval, document management, automatic classification, application integration, image acquisition, multi-language text extraction.

\section{An Outline of CAIRN Systems}

Information, in any format, is a strategic resource and it should be available to the user from the desktop as if it were located in a personal library. In other words, any user needs easily accessible information and tools to exploit it. In this context, the popularity of the Internet has caused an exponential increase in the number of people who use on-line text but the quality and usefulness of documents varies widely and web search tools are characterised with extremely low precision. In other words, an annoying consequence of information retrieval from the Internet is the fact that there is a vast amount of garbage that surrounds any useful information. It is even worse to put the users through the annoying process of separating interesting information from garbage, every time they look for (some kind of) information that they had already found in the past. Thus it could be very useful if the users could include the interesting information in a personal library and avoid repeatedly separating garbage from useful information. Such information should be easily accessible and digestible. When the users have a question, they should have the means (the software system etc.) to find in their personal library their own previously collected related material and support their own answers / decisions / research.

A few years ago, an outline / definition of the term information could include articles, technical specifications, manuals, personal comments, notes and, generally, any information that could be represented as ascii (plain) text, popular word processor formats and html files. Over the years, there has been a number of powerful text retrieval software / tools for indexing and retrieving information, without supporting the whole range of information (as it was defined so far). Such examples include the SMART system [2, 34], the INQUERY system [8] and the SEFT public domain software [21].

A well known, crucial requirement was to increase the value of the stored information. As 
an example, Karanikolas and Skourlas [15] discussed the first framework of a useful Computer Assisted Information Resources Navigation (CAIRN) architecture. It was designed for storing and organizing in the personal library a whole range of information (as it was defined so far) and introduced the possibility for the user to access information using Natural Language queries. Such a system could hide information management procedures and its users needn't have had any knowledge in librarian's tasks (e.g. Cataloguing, indexing, abstracting). All these tasks were extremely time and money consuming activities.

During these years, commercial tools appeared, which offered various possibilities for organizing and retrieving information from data sources. We can mention NEXTPAGE [49], HYPERION [41] and AUTONOMY [38]. NEXTPAGE is an antecedent of Folio that simplifies the management of documents' version. Sirsi, provides the HYPERION Digital Media Archive for building, storing, and maintaining collections of digitally captured material. AUTONOMY [38] (redeem Verity) provides applications for making content from the enterprise, entirely searchable. The phrase "content from the enterprise" is assumed as content from corporate networks, intranets, local data sources and information from the desktop.

Today, end users consider that information is almost anything that they can have on their hard disk. The GOOGLE DESKTOP [40] reflects this perception of information. It offers easy access to information on the local storage and from the web. It is a desktop search application that provides full text search over emails, document files, digital media and web pages that the users of a computer have already viewed. By making the computer searchable, Google Desktop prevents the need for manual organization of files, emails and bookmarks.

This fact motivated us to investigate and describe a new family of CAIRN architectures.

The requirements for the proposed architecture are the following:

- It should offer the ability to collect information from different resources: WEB-based documents, bibliographies in machine-readable form, ASCII (plain) text, Office Automation documents (e.g. .doc and .rtf files). It should also support the storage of personal notes, personal research reports etc. Other potential sources of information are: pdf documents, email documents, digitized pictures, images, video captures, etc.

- Information should be accessed using both Natural Language and Field-Based queries (Queries-By-Example approach - QBE).

A domain that could benefit from the application of systems that conform to the new architecture is the medical domain [16]. Medical information can cover patient discharge letters, Computer Tomography (CT), MRI and Ultrasound images, etc.

In Section 2 we present the new CAIRN architecture. A prototype tool is described in Section 3. In Section 4 some technological solutions are discussed. In Section 5 our evaluation is presented and discussed, and eventually future activities are briefly given in Section 6 .

\section{A Modular CAIRN Architecture}

A long list of concrete capabilities must be embodied in order to implement such a system. The architecture of CAIRN systems is depicted in Figure 1.

The concrete capabilities of the implementation of a CAIRN system are:

\subsection{Text Indexing Capabilities}

Text Indexing capabilities can be based on the Vector Space Model (VSM) [20], utilizing algorithms for word stemming and the usage of Inverted Files. Other models for Text Indexing and Document Representation could also be used. However, it has been proven that the VSM is the most popular model. In a recent review [3], the number of systems that use the VSM for Document Representation are twelve (12) of eighteen (18) systems that the authors have knowledge of their technical details. According to the VSM model a vector of $k$ elements represents each document, where each element is the weight of one of the selected indexing terms (word stems). For example, the $i$-th document is represented by:

$$
D_{i}=\left\{t_{i 1}, t_{i 2}, \ldots, t_{i k}\right\}
$$




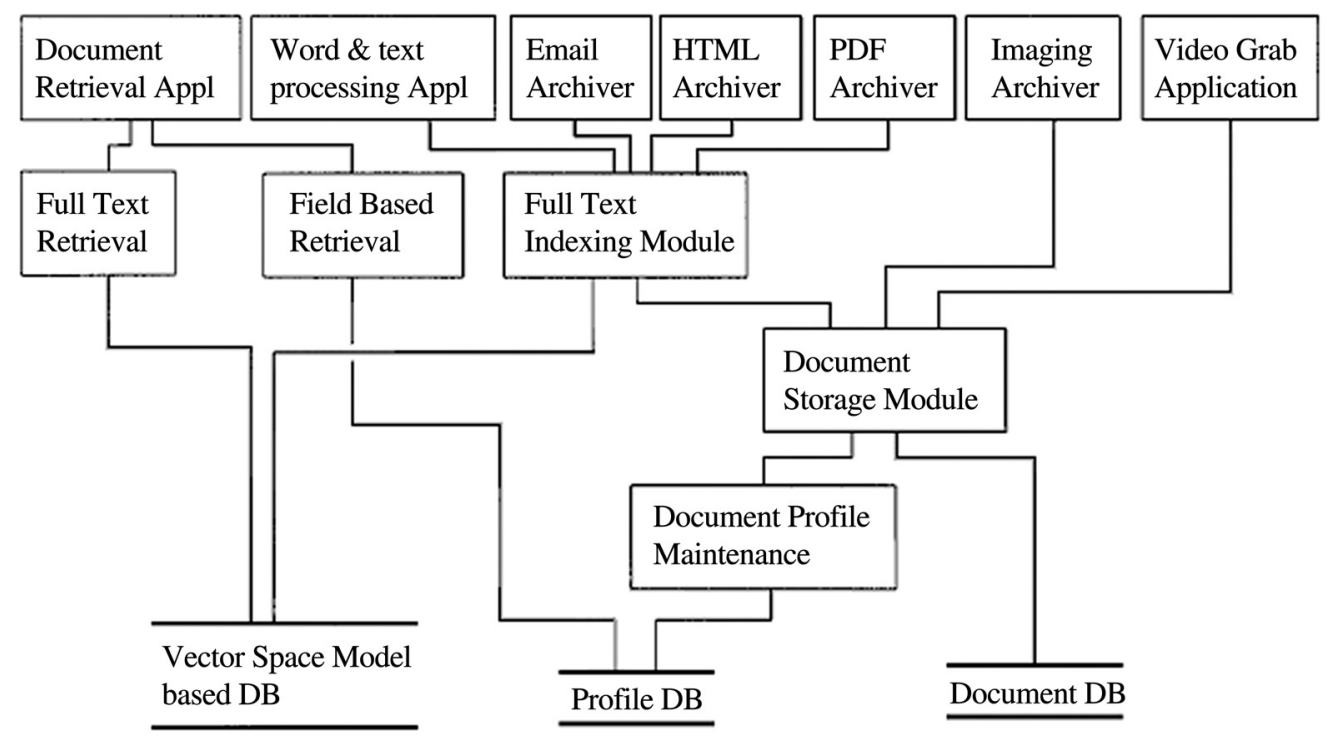

Fig. 1. The architecture of CAIRN systems.

where $t_{i j}$ is the weight of term $j$ in document $i$ and $k$ is the number of the selected weights for indexing word stems (terms). The system must be able to stem words $[14,27,35]$ for every supported language. By using stems instead of words for indexing textual documents we achieve a significant reduction of the overhead and also an improvement to the recall of documents. The usage of Inverted Files $[6,34]$ is needed for storing the locations and the weights of every stem selected for indexing textual documents. Text Indexing capabilities should be implemented in the corresponding Full Text Indexing Module.

\subsection{Still Image Acquiring Capabilities}

CAIRN systems must be able to handle scanners and digital cameras in order to acquire images. The problem raised in this case is that the CAIRN systems must be able to "understand" and communicate with the driver of each available scanner and digital camera. This requires a vast amount of application development and maintenance for supporting each new released image device. To overcome this difficulty, CAIRN systems must be able to use some abstract (standard) communication with image devices and avoid the need for constant revisions for supporting new and revised devices. The still image acquiring capability must be implemented in the Imaging Application of a CAIRN system.

\subsection{Image Acquiring from Video Sources}

With "image acquiring from Video sources" we address the capability to connect CAIRN systems to video sources and grab images either by user intervention or in some automated way. In the latter case the system should offer an interface where the user is able to define the time interval between two consecutive grabs and start the acquiring process. This process will consist of grabbing a separate image for every multiple of the defined time interval and will stop when the user decides to. The problem raised in this case is similar to the problem raised for still image acquiring and there is a need for an abstract (standard) communication between CAIRN systems and Video acquiring devices (usually Video frame grabbers). The image acquiring from video sources capability must be implemented in the Video Grab Application constituent of a CAIRN system.

\subsection{Image Storage}

The storage of acquired images is an obvious necessity. There are a lot of image formats and CAIRN systems must be able to support a wide variety of them. We can mention: PNG (Portable Network Graphic), TIFF 
(Tagged Image File Format), JPEG (Joint Photographic Experts Group), WMF (Microsoft Windows Metafile), PCX (PC Paintbrush File format), Bitmap (Microsoft Windows Bitmap) and GIF (Graphics Interchange Format). However, CAIRN systems must support at least the most widely acceptable and most appropriate for cross (software and hardware) platform image archiving. Since TIFF format permits both MSB ("Motorola") and LSB ("Intel") byte order data to be stored, it is a highly flexible and hardware-platform-independent format, which is also supported by numerous imageprocessing applications. Moreover, because developers of printers, scanners and monitors designed it, TIFF is a very rich space of information elements for colorimetry calibration, etc. The last TIFF specification [54] is a definition of single and multi page image file format. It supports Black/White, Grayscale, Palette-color and RGB Full color images. It also has support for CMYK, YCbCr and $\mathrm{L}^{*} \mathrm{a} * \mathrm{~b} *$ images. It has a trenchant documentation and great acceptance. Consequently, TIFF storage is an obligatory capability for CAIRN systems. Another candidate for image storage is JPEG. JPEG $[11,12]$ is a definition of single page image file format with great acceptance for storage of photographs. It is used for continuous tone image compression (truecolor (24bit) and grayscale (8bit)). The lossy class (there is also a lossless class) of JPEG provides substantial compression without significant degradation of the reconstructed image. The only exception occurs in case of artwork with sharply defined lines.

The storage of video information is another interesting topic. The most appropriate format is the MPEG (Moving Picture Experts Group) standard/format. Under the name MPEG, there is a family of international standards that are defined for coding audio-visual information in a digital compressed format. The MPEG family (MPEG organization [48]) of standards includes MPEG-1, MPEG-2, MPEG-4, MPEG7 and recently MPEG-21. MPEG-1 standard has enabled storage of video on CD-ROMs. MPEG-2 [5] standard has broadened the storage of video on DVDs and also made the Digital Television possible. MPEG-4 [19] and the subsequent ones are more oriented to more semantic representations and therefore, they are of no interest for CAIRN systems. Another alternative/candidate for the storage of Video information could be the Motion JPEG 2000 (MJ2). MJ2 [46] defines a file format for motion sequences of JPEG 2000 images. It also supports storage of associated audio. MJ2 does not involve inter-frame coding and each frame is coded independently using JPEG 2000.

\subsection{Image Compression / Decompression}

Since the space needed for storage of images is a very significant factor, there is a necessity for compressing images. The CCITT Group 4 compression/decompression algorithm [13] is a standard for compressing/decompressing black/white images and it is supported by TIFF format. For color pictures, the Lempel, Ziv and Welch (LZW) algorithm [37] is a lossless compression technique supported by TIFF. Unfortunately, to use this technology, an LZW license agreement is necessary (the patent is held by Unisys), regardless of the library used, or even if the code is written from scratch. Another alternative could be the JPEG lossy compression that is also supported by the TIFF file format. The only drawback with JPEG compression in TIFF is the loss of information. Fortunately, an alternative, non-proprietary compression scheme has been proposed, based on the ZLIB/Deflation stream. It is a lossless compression standard derived from LZ77. Adobe has recently added a deflate compression option for TIFF, referred to as Zip-in-tiff compression. Deflate [4] generally yields better results compared to LZW and without any loss of information.

The latter two capabilities should be implemented in both Imaging and Video Grab Applications.

\subsection{Application Integration}

The environment which is offered by CAIRN, is a multi-type and multi-format archiving environment. It must provide some degree of applications' collaboration. For example, the retrieval module of a CAIRN system must be able to present an image-based document selected by the user. To achieve this, the Document Retrieval module (application) must invoke the relevant Imaging Application and order this application to open the user-selected document. 
Another example of application integration is faced for printing a document without even presenting the native application to the user. The integration in this case implies invoking the native application in an invisible mode, ordering this application to open the user-selected document, ordering this application to print the document and finally ordering the native application to exit. For application integration, the applications can be seen as objects, exposing interfaces that can be invoked by other applications. Here, the term object has a different meaning / interpretation from the well known one in the case of the object-oriented programming languages. It is used to express reusable software entities deployed as binaries, and is also known as components. The significance behind "exposing interfaces" to the world is that these interfaces are the "sockets" into which other components and applications can plug. This behavior contributes to the reusability of software components. The exposed interfaces of components provide services that can be requested by clients. Thus, components are (such components act like) Servers. The Clients are applications that can plug into the interfaces exposed by Servers and, consequently, they can access their services by issuing requests that contain information about the target component (Server), the operation needed, the parameters for the operation (if any) and any optional request contexts. These requests cause services to be performed.

\subsection{Email Archiving}

Since email is one of the most utilized methods for personal and business communication, CAIRN systems must be able to collect and archive the information that emails carry. This information can be the email body, but also the documents transferred as email attachments. Thus, CAIRN systems have to communicate with the Email clients and gain access to the incoming messages. However, there are more than one Email clients and, consequently, CAIRN must be able to communicate with all or most (the most proliferated) of them. The problem raised in this case is similar to the aforementioned problem for supporting new hardware devices for image acquisition. The difference here is that there is the requirement for supporting new software products and releases, instead of new and revised hardware devices. In this case, the solution is the same, i.e. to use some abstract (standard) communication between CAIRN systems and email clients. The capabilities for standard communication between CAIRN and email clients, gaining access to the incoming messages and archiving the information that these messages carry should be implemented in the Email Archive module.

\subsection{Extraction of Multi Language Text}

Extraction of multi-language text capability refers to the necessity for distinguishing the different languages used in the body of each textual document and marking down the text every time there is a change in the language used. The way that this capability is accomplished is dependent on the source of information. For example, if the source is a word processor document, the CAIRN system takes advantage of application integration to invoke the native application in invisible mode, orders this application to open the source document, orders to save the source document as an RTF (Rich Text Format) temporary file and finally orders to exit. Then, an RTF reader [52] elaborates the RTF temporary file and extracts the Multi-National-Text (on the base of \lang tags found on the temporary RTF file). In case that the source is an HTML file, CAIRN system is based on HTML tags and/or Unicode characters [10] instead of usual characters for finding the language changes. Examples of such HTML tags are $<$ meta http-equiv="Content-Type" content $="$ text $/ \mathrm{html}$; charset=windows $-1253 ">$ and $<$ meta http-equiv="Content-Language" content="greek" $>$. An example of a Unicode character sequence is \&\#932;\&\#959;\&\#954;\&\#945; \&\#955;\&\#973;\&\#964;\&\#949;\&\#961;\&\#959; and represents the greek phrase "То к $\alpha \lambda u ́ \tau \varepsilon \rho о$ ".

The capability for Multi-National-Text extraction is a capability that should be present at least in the Word and Text Processing Application, PDF, HTML and EMAIL Archive.

More than the above mentioned capabilities and the modules that should provide them, the CAIRN Architecture is based on some other modules that support the operations of Text and Field-Based Retrieval, Document Storage and Document Profile Maintenance. 


\subsection{Full Text Retrieval Module}

This module should provide a graphical user interface where the users will be able to submit their queries using natural language. Natural Language queries allow the users to enter a prose statement describing the information they want to find. The similarity of a submitted query against the documents of the system must be calculated with some similarity measurement function [34]. A well known such function is the Nearest Neighbor Matching function [22]. This is a cosine-based similarity measure between a document and a query or between two documents.

$$
S\left(D_{i}, D_{j}\right)=\frac{\sum_{r=1}^{k} t_{i r} t_{j r}}{\sqrt{\sum_{r=1}^{k} t_{i r}^{2} \cdot \sum_{r=1}^{k} t_{j r}^{2}}}
$$

A small improvement to the cosine similarity for a medical data collection has been presented by an earlier CAIRN prototype [15]. Other models for Information Retrieval $[3,36]$ could also be used.

\subsection{Document Profile Maintenance Module}

This module should provide a graphical user interface where the users will be able to provide the values of fields that characterize documents. The most interesting idea that should be implemented in this module is that each field can be able to hold more than one value (an infinite number of values) and that each value can be composed of one or more attributes. In this way a field can have a single value, a list of values or a table of values. In the latter case the rows of the table represent the multiple values and the columns represent the attributes of each value (row).

\subsection{Field-based Retrieval Module}

This module should provide a graphical user interface where the users will be able to impose restrictions on one or more fields, in order to retrieve the documents they are interested in. A new demand is that the user must be able to provide two (or more) values for a field and demand that the retrieved documents must contain both (all) provided values. This is a consequence of the ability of the Document Profile Maintenance module to permit more than one value in each field.

\subsection{Document Storage Module}

This is a mediator that defines an internal file name (never used by users), does the actual storage of documents and invokes the Document Profile Maintenance module.

Some of the above capabilities are dependent on technologies that are available in the Operating Systems. Some other capabilities are defined in an abstract level and consequently are independent from the Operating Systems. The ideal solution for cross platform development of CAIRN systems would be to have the same technologies available in most desktop operating systems (Windows, Mac OS, Linux, Solaris). However, this is not a rule. The capabilities defined on an abstract level, and consequently available for implementation in every operating system, are: text retrieval (Inverted Files, Vector Space Model, Nearest Neighbor Matching, etc), image storage, image compression/decompression, Multi-National text extraction. The problem arises for the rest of the needed capabilities. Still image acquiring, Image acquiring from Video sources, Application Integration and finally Email archiving are capabilities dependent on the technologies available in the desktop Operating Systems. These technologies have neither common standards nor common APIs and, consequently, different means must be used for implementing the latter capabilities.

Our effort to design CAIRN Architecture has been complemented with an attempt to build a CAIRN system prototype. We have built the CAIRN system prototype for the Windows Operating System (OS). Next chapter explains the technologies used for implementing the latter (not defined on an abstract level) capabilities in the CAIRN system prototype. 


\section{A CAIRN System Prototype under Windows}

The technologies we have used in the development of CAIRN System prototype, which are tightly coupled with the Windows OS are TWAIN [55, 56, 57], OLE Automation [1], MAPI [45], Video for Windows [60]. TWAIN is an API, maintained by an organization that offers a common way for acquiring images from scanners and digital cameras. This API is available only for Windows and Mac OS. OLE Automation stands for Object Linking and Embedding Automation and is available only for Windows Environments. The same stands for Video for Windows. MAPI is an abstract level of communication between applications and Mail clients (e.g. Eudora, Outlook).

\subsection{TWAIN}

In the CAIRN system prototype, the Imaging Application is a TWAIN-enabled application. TWAIN (TWAIN Version 1.6 [56], TWAIN v1.7 [57]) is an API, maintained by the TWAIN Working Group (TWAIN Organization [55]) for simplifying the difficulty of connecting scanners and personal computers. TWAIN is an image-capture API for the Microsoft Windows and Apple Macintosh operating systems. That API was introduced in 1992. TWAIN requires three software elements (and hardware) working together to enable image acquisition. These are Host Application, Data Source and Source Manager. The Host Application presents a File menu, with a "Select source" and an "Acquire" menu items for choosing an image-acquisition device and obtaining an image from that device respectively. In response to the user selecting one of those menu items, the application sends messages to TWAIN. When acquiring an image, TWAIN sends messages to the Host Application that the application handles in its messagehandling loop. The Data Source is a driver that controls a specific image-acquisition device and is written by the device's manufacturer to conform with the TWAIN specification. TWAIN places the graphical user-interface to control the device in the Data Source driver instead of the Host Application. This makes it easy to implement a still image acquire application, but it presents some difficulties for other operating systems.

\subsection{OLE Automation}

OLE Automation [1] is an object technology coming from Microsoft. OLE Automation must not be confused with OLE Object Embedding, OLE Object Linking and OLE In-place Activation. OLE Automation is the formal interprocess communication technique for the Windows Operating System. OLE Automation is based on fundamental object-oriented programming ideas. It provides the infrastructure and guidelines that let an application share objects called automation objects (also called automation servers). Other applications, called $a u$ tomation controllers, communicate with the application by reading or setting the shared objects' properties or by calling the shared objects' methods. Visual Basic is an example of a wellknown automation controller. OLE Automation defines standard ways in which an automation object publishes information about what methods and properties it supports. Automation controllers provide browsers so programmers can read this information. An impressive example of support for OLE Automation is Microsoft Office. Almost every feature of Word, Excel, and PowerPoint is accessible to end-user programming. Programs that control Office can be written with Visual Basic [25, chapter 22] or with a special version of Visual Basic included in Office called Visual Basic for Applications. At this point, it is appropriate to make it clear that not only the MS development tools are able to create OLE Automation controllers and OLE Automation servers. In other words, using OLE Automation, someone can integrate two or more applications in a way that appears seamless to the user. Working with OLE Automation objects is very similar to working with objects in the application itself. That means that the $a u$ tomation controller retrieves some object and the object's properties from an OLE Automation server and applies the object's methods as if they were in the application itself.

An interesting usage of OLE Automation in CAIRN System prototype is for creating Reach Text Format files from .doc files (without user intervention) and consequently parsing the RTF files to extract multi national text (text pointing out language changes). Multi national text is convenient for bilingual and multilingual full 
text indexing. In this case the Full Text Indexing module (of the CAIRN system prototype) is the OLE Automation controller, while MS Word is the OLE Automation server.

Another interesting usage of OLE Automation is for the invocation, from the Document Retrieval Application (of the CAIRN system prototype), of the native application for each document type. In case of a TIFF file we face the case of communicating the Document Retrieval Application (OLE Automation controller) with the Imaging Application (OLE Automation server) of the CAIRN system prototype.

\subsection{Video for Windows}

Video for Windows (VfW) [60] is a complete system for maintaining video on Microsoft Windows. In the beginning, VfW was a collection of 16-bit windows utilities, dynamic link libraries and other components. Version 1.0 of VfW was released in November 1992. It was followed by version 1.1. An additional letter characterizes the following versions. The most recent version of VfW 1.1 for the operating system Windows 3.x is 1.1e. Microsoft has released a 32-bit version of VfW for the operating system Windows 95. From the Microsoft's Internet site, VfW 1.1e can be downloaded in two deployments: Runtime or Development Kit. In contrast with Windows 3.x, Windows 95 includes VfW.

A VfW capture driver usually accompanies video capture cards supporting VfW. This driver exports some predefined functions and dialog boxes that every VfW compliant application is able to invocate.

The VfW API is based on the creation of a Capture Window and the connection of this dialog box with a VfW capture driver (the corresponding capture driver for the Capture card). Then, with the submission of messages towards the Capture Window, the VfW API permits the accomplishment of actions, such as capturing of a still image, recording a video clip (AVI), projection of video signal through the video capture window (preview), projection of video signal directly to the screen without the intervention of the computer (overlay), control of the attributes of video signal (System:
PAL/SECAM/NTSC, Image Dimensions, Image Format: 24-bit RGB/16-bit RGB/8-bit Palletized), etc. There are also messages that are used to declare a callback function. This callback function is defined in the user's application and is activated automatically by VfW every time a specific event happens on the video streaming.

The Video Grab Application of our CAIRN system prototype is based on VfW and it is presented in the next figure:

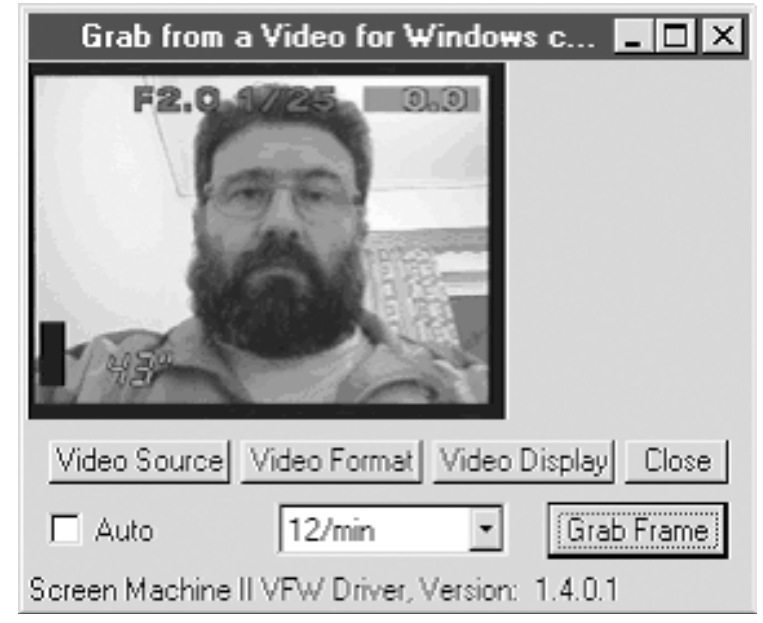

Fig. 2. The Video Grab Application of CAIRN system prototype.

This application implements the image acquiring from Video sources capability and it is complementary to the TWAIN-enabled application, since it permits capturing one or a sequence of still images from a video source, instead of a scanner or a digital camera.

\subsection{Messaging Application Programming Interface (MAPI)}

Messaging (Mail) Application Programming Interface (for short MAPI) [45] is a standardized set of $\mathrm{C}$ functions placed into a Dynamic Link Library (DLL). These functions permit any Windows application to become "mail-enabled". With "mail-enabled" we mean that the windows applications can send and receive emails. The truth is that the Windows appli- 


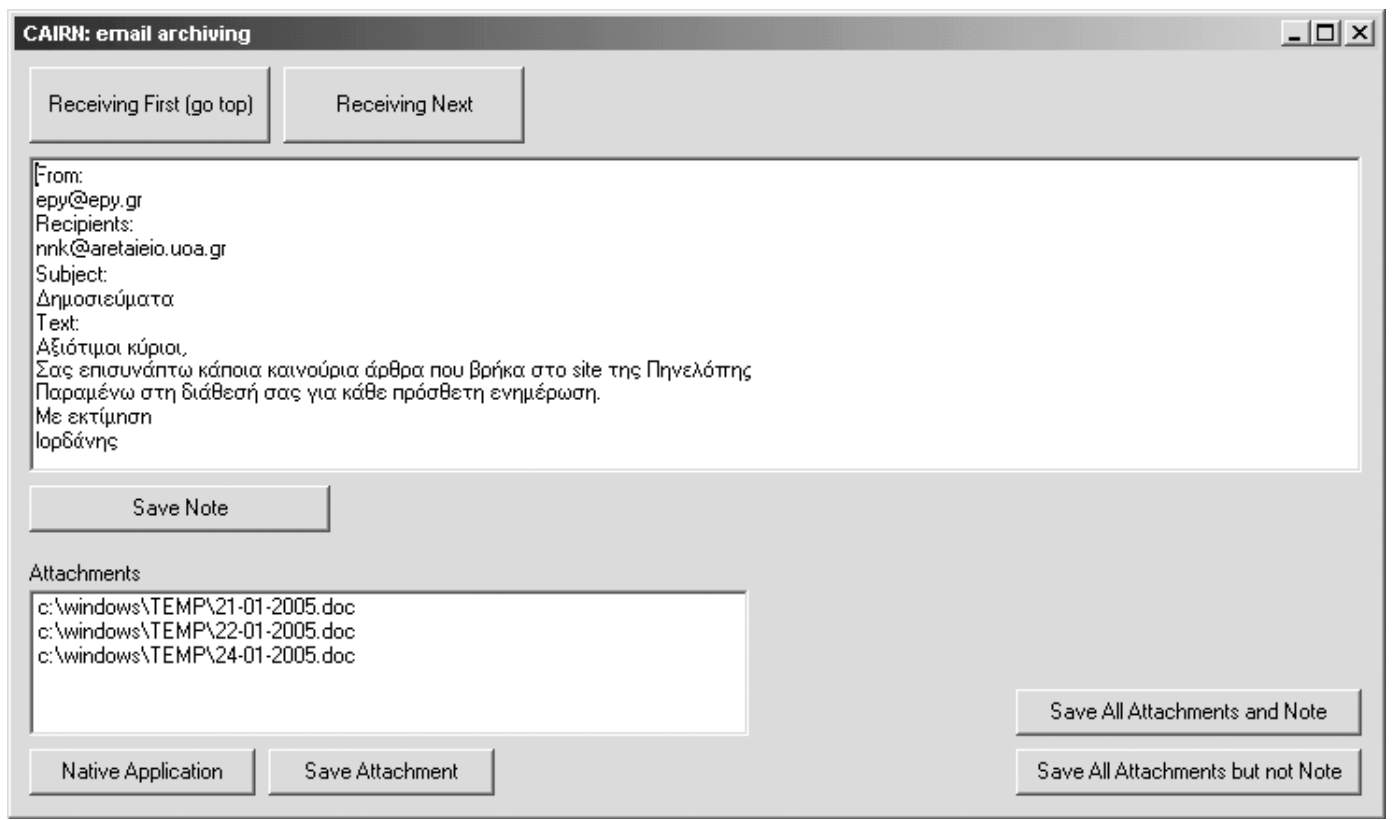

Fig. 3. The Email Archiving module, of the CAIRN system prototype.

cation submits emails to the Mail client and receives emails from the Mail client and does not communicate with the Mail Server. There are actually two sets of functions defined for the complete MAPI library. The first twelve functions are known as Simple MAPI, while the remaining functions constitute the Extended MAPI. The functions of Simple MAPI are enough for sending and receiving emails.

The need for email archiving can now be easily accomplished. Figure 3 presents the Email Archiving module, of the CAIRN system prototype based on MAPI, for archiving data of emails.

The navigation through emails is accomplished with the buttons "Receiving First" and "Receiving Next". For each message, the user can see the mail body and a list of attached files. For each attachment, the user can activate the Native Application. The archiving can be done separately for the email body and each attachment, but it can also be done collectively.

\section{Transition to UNIX / Linux}

This section introduces some technologies that could be used for the implementation of the CAIRN Architecture in the Linux and Unix OS.

\subsection{SANE}

SANE [53] is an acronym for the phrase "Scanner Access Now Easy". It is an applicationprogramming interface (API) that provides access to any raster imaging hardware device (scanners, video and still cameras, frame grabbers, etc.). The SANE API is public domain and its discussion and development is open to everybody. The current source code is written for UNIX (including GNU/Linux) and is available under the GNU General Public License. Like TWAIN, SANE allows the creation of just one driver per image acquisition device, rather than one driver for each combination of device and application. However there are reasons that do not permit the straightforward replacement of TWAIN with SANE. One of the reasons why TWAIN is not SANE, is that TWAIN puts the graphical user-interface used to control the device in the (data source) driver instead of the application. From the user's point of view this means that the same application represents a different scanning interface whenever a different TWAIN data source (device) is used. From a technical point of view, this makes TWAIN unsuitable for UNIX / Linux since the TWAIN drivers are interwoven with a particular GUI (currently MS Windows or Macintosh) while the applications developed for UNIX / Linux 
can have one of a variety of interfaces (console/text mode, GNOME, KDE, X11 or Motif Windows). In contrast, SANE clearly separates device controls from their representation in a user-interface. As a result, SANE has no difficulty supporting a variety of interfaces, ranging from command-line interfaces to any Graphical user-interface and the access to the device can happen locally or over the network.

For the above-mentioned reasons, the development of a still image-acquiring module of a system, respecting the CAIRN Architecture and designed for UNIX and Linux OSs, must be extended to the development of a scanning interface sub-module. This sub-module will be presenting the appropriate, for the used GUI window manager (GNOME, KDE, Motif, X11), interface to the user and on the background it will be using SANE negotiations for activating/deactivating the available scanning features, depending on the selected scanner.

\subsection{Bonobo}

BONOBO [39] is the GNOME architecture for creating reusable software components and compound documents. Bonobo components help reducing the complexity of applications by reducing the amount of information a programmer needs to know about the system. This is achieved by making each software component implement a very well defined interface. Bonobo components also enable programmers to build larger, more complex applications by gluing different components together into bigger applications. The key point behind component programming is in the "interfaces" that components export to the world. Each one of these interfaces is a "socket" other components and applications can plug into. These interfaces are defined in terms of CORBA interfaces, specified in the CORBA Interface Definition Language. According to this architecture, the clients submit requests asking for some services. These requests are transmitted to the object request broker (ORB) who is responsible for forwarding the requests to the adequate server (service provider).

For the Application Integration needed, the modules of CAIRN must be implemented with respect to the Bonobo architecture, i.e. as objects that export interfaces. For example, the Imaging module of CAIRN must export an interface that permits: its invocation, the loading of designated documents, etc. There is an obvious question. What about the ready made native, for the archived documents, applications? The answer is that Bonobo architecture is a proliferated architecture and it is supported by most shelf applications. For example, Gnumeric Spreadsheet, GNOME postscript viewer and GNOME PDF viewer are Bonobo compatible.

\subsection{Video for Linux}

Video for Linux [59] is the corresponding Linux technology to the Video for Windows technology that exists in the Windows OS. Video for Linux (v4l) is the original capture/overlay API of the Linux kernel. It appeared late, in the 2.1.x development cycle of the Linux kernel. Video for Linux Two (v412) is the second generation of the video for Linux API. It was integrated into the standard kernel in 2.5.x. V412 is a thorough overhaul of the API with respect to Video for Linux.

It is obvious that the Image acquiring from Video sources module, of a CAIRN system designed for Linux, must be implemented taking advantage of v412 API.

\subsection{Mail Application Programming Interface for Linux}

Unfortunately, the famous Mail clients for the Linux OS (Mozilla Thunderbird [47], Ximian Evolution [61]) do not completely support MAPI. In general, under Linux, the available libraries communicate directly with Mail Servers instead of communicating with Mail Clients. On the other hand, communication with e-mail Servers is a common experience in the Linux domain. More precisely, a vast number of scripting languages support directly submitting and receiving emails [7, chapters 9-12], [26, chapter 22], $[9$, chapter 15$]$. There are also some tutorials and related libraries for e-mail communications $[9,23,24,43,44,50,51]$. The exact definitions for communications with Email Servers are defined respectively by Requests for Comments $[28,29,30,31,32]$. In order to implement an application similar to the application presented 
in section 3.4, we believe that it is enough to replace the MAPI requests with the code that respects Post Office Protocol - Version 3 [29].

The next chapter presents the first evaluation based on our effort to design and implement the CAIRN prototype.

\section{An Evaluation Based on the Design and Implementation of CAIRN Prototype}

In the previous sections we have discussed how the current technological environment can support the development of software that handles unstructured information and CAIRN systems for most desktop operating systems. Unfortunately, the technologies used for the different desktop operating systems are not always compatible and, in specific cases, we have to adapt different technologies for different operating systems.

Some results of the scientific research in Information Retrieval (e.g. algorithms for text indexing, text retrieval, word stemming, and the use of specific similarity measures) and the standards established by international organizations of standardization (e.g. CCITT Group 4 compression) can usually form a basis for cross-platform implementation. However, proprietary, de-facto commercial standards must be considered for possible implementation / integration into such a tool. Some of them could be easily supported independently from the platform, the operating system and the tools of software development that each developer uses.

The existence of open source code also facilitates cross-platform implementation. As an example, we can mention the incorporation (integration) of free JPEG software (source code) of the JPEG Group [42] with minor changes into the CAIRN prototype.

There are also serious problems in supporting / integrating into CAIRN tools some commercial standards / products when in different operating systems we encounter different technologies for the achievement of the same objectives. Thus, for the communication and the utilization of one application by another, a Linux/Unixbased tool could use Bonobo / CORBA and an MS Windows-based system could use OLE Automation / COM / DCOM [1,33]. Another example is that under Linux the applications could communicate directly with mail servers, whereas under Windows the applications could communicate with the Mail client, in order to read and submit email messages.

Proportional differences exist also in the capture of still image and Video signal. In certain cases, this is justified from the philosophy of (or the inheritance from predecessor) operating systems. For example, in Linux there is no standard interface and it is considered almost mandatory that the application software (the tool) must have access to a scanner that is found in another (remote) system. On the contrary, in the MS Windows there is usually only one interface and the support of remote image acquiring devices is not essential for the developer.

A common sense strategy to overcome the barrier of using different technologies for implementing the same product for different operating systems is to develop abstract tools (technologies) that combine only the best features of the different existing technologies. To clarify our point of view we can briefly discuss the perspective that the TWAIN working group proceeds, in order to expand TWAIN's portability to include UNIX [58]. More precisely, they are redesigning TWAIN in order to operate as a front-end and use SANE as its back-end in the UNIX environments. The application developer will still have the ability of "Linking Images With Applications" easily, which is the TWAIN's whole purpose. The TWAIN group believes that most applications will rely on the TWAIN driver's GUI, because the amount of code required is reduced. On the other hand, SANE is an established standard for UNIX and has the benefit of years of Open Source development work. Therefore, TWAIN does not intend to replace SANE and "reinvent" solutions to fundamental device communication problems.

\section{Future activities}

In the future, we intend to extend the CAIRN Architecture in order to adopt new sources of information (e.g. DICOM and XML) and offer to the user more refined tools for retrieving and organizing information. We plan to support and integrate into our tool an automatic document 
classification concept / possibility. Hence, the classification of new documents could be based on their similarity with the existing ones (of a "training" set). Such an Instance-based learning method assumes that similar documents must be classified in the same category or share the same classification code [17]. In a complementary way, the classification of documents could be based on naive rules inducted from training sets of pre-classified documents [18]. Future extension will elaborate the incorporation of ontologies and lexical tools for text disambiguation. We also plan to proceed with some experimentation with Cross Language text collections, especially for medical texts.

\section{Acknowledgments}

This project is co-funded by the European Social Fund and National Resources - (EPEAEKII)-ARXIMHDHS.

I would like to thank my colleague, Professor Christos Skourlas, for his continuous support and the discussions we have had over the past eighteen years.

\section{References}

[1] K. BRockschmidT, Inside OLE. Microsoft Press, Printed in the USA, second edition, 1995, ISBN 1-55615-618-9.

[2] C. BuCKLEY, Smart ver. 11.0, 1992. ftp://ftp.cs.cornell.edu/pub/smart

[3] G. CANForA, L. CERUlo, A Taxonomy of Information Retrieval Models and Tools. Journal of Computing and Information Technology, 12 (2004), pp. 175-194.

[4] A. FeldSPAR, An Explanation of the Deflate Algorithm, 1997.

http://www.zlib.net/feldspar.html

[5] C. FogG, MPEG-2 FAQ: Questions that Should Be Frequently Asked About MPEG, 1996. http://www.mpeg2.de/doc/mpeg2faq/index .htm

http: //www.mpeg2.de/doc/mpeg2faq/faq_v38 .htm

[6] M. J. Folk, B. ZoELLICK, File Structures. AddisonWesley, Printed in the USA; 1 st edition, 1987, ISBN 0-201-12003-8.

[7] J. GoERZEN, Foundations of Python Network Programming, APress, USA, ISBN 1590593715.
[8] HARMAN, Overview of the fourth text retrieval conference (TREC-4).

http://trec.nist.gov/pubs/trec4

loverview.ps.gz

[9] S. HuGHES, PHP Developer's Cookbook, Sams Publishing, Second Edition, 2001, ISBN-10: 0-672-32325-7.

[10] ISO 10646-1, Information technology - Universal Multiple-Octet Coded Character Set (UCS) - Part 1: Architecture and Basic Multilingual Plane, 1993.

[11] ISO/IEC IS 10918-1, ITU-T T.81, Digital Compression and Coding of Continuous-tone Still Images, Part 1: Requirements and Guidelines.

[12] ISO/IEC IS 10918-2, ITU-T T.83, Digital Compression and Coding of Continuous-tone Still Images, Part 2: Compliance testing.

[13] ITU-T Recommendation T.6, Facsimile coding schemes and coding control functions for group 4 fascimile apparatus, (c) ITU 1988, 1993.

[14] T. Z. Kalamboukis, Suffix stripping in Greek. Program, 29 (1995), pp. 313-321.

[15] N. N. Karanikolas, C. Skourlas, ComputerAssisted Information Resources Navigation. Medical Informatics and the Internet in Medicine, 25 (2000), pp. 133-146.

[16] N. N. Karanikolas, C. SKourlas, Shifting from Legacy Systems to a Data Mart and ComputerAssisted Information Resources Navigation Framework. Proceedings of the $5^{\text {TH }}$ International Conference On Enterprise Information Systems $5^{T H}$ ICEIS, (2003) Angers, France.

[17] N. N. Karanikolas, C. SkOURlas, A. Christopoulou, T. Alevizos, Medical Text Classification Based on Text Retrieval Techniques. Proceedings of the $1^{S T}$ International Conference on Medical Informatics \& Engineering $-1^{S T} M E D I N F$, (2003) Craiova, Romania. Craiova Medicala, volume 5, supplement 3, pp. 375-378.

[18] N. N. Karanikolas, C. Skourlas, Naive Rule Induction for Text Classification Based on KeyPhrases. Proceedings of the $6^{\text {TH }}$ International Conference on Data Mining, Text Mining and their Business Applications, (2005) Skiathos, Greece. WIT Transactions on Information and Communication Technologies, volume 35, Data Mining VI: Data Mining, Text Mining and Their Business Applications, WIT Press.

[19] R. KoEnEn, Overview of the MPEG-4 Standard, 2002.

http: //www . chiariglione.org/MPEG

/standards/mpeg-4/mpeg-4.htm

[20] G. KowALSKI, Information Retrieval Systems. Theory and Implementation. Kluwer Academic Publishers, Printed in the USA, 1st edition, 1997. 
[21] O. D. Kretser, A. MofFAt SEFT, A Search Engine for Text. Software Practice and Experience, 34 (2004), pp. 1011-1023.

http://www.cs.mu.oz.au/ alistair

/abstracts/dm04spe.html

http://www.cs.mu.oz.au/ alistair

/abstracts/dm00:acsc.html

http://www.cs.mu.oz.au/ oldk/seft/

[22] D. LuCARELla, A document retrieval system based on nearest neighbour searching. Journal of Information Science, 14 (1988), pp. 25-33.

[23] D. Marshall, Using Perl to Send Mail http://www.cs.cf.ac.uk/Dave/PERL /node180.html

[24] D. Marshall, Receiving Mail (POP) http://www.cs.cf.ac.uk/Dave/PERL /node181.html

[25] G. Perry, Sams Teach Yourself Visual Basic 6 in 24 Hours, Sams Publishing, 1999.

[26] C. PIERCE, Sams Teach Yourself Perl in 24 Hours, Sams Publishing, 1999, ISBN 0-672-31773-7

[27] M. F. PORTER, An algorithm for suffix stripping. Program, 14 (1980), pp. 130-137.

[28] RFC 821: SMTP

http://www.faqs.org/ftp/rfc/pdf /rfc821.txt.pdf

[29] RFC 1939: POP v 3

http://www.faqs.org/ftp/rfc/pdf /rfc1939.txt.pdf

[30] RFC 1730: IMAP version 4 http: //www.faqs.org/ftp/rfc/pdf /rfc1730.txt.pdf

[31] RFC 2060: IMAP version 4.1 http: //www.faqs.org/ftp/rfc/pdf /rfc2060.txt.pdf

[32] RFC 2683: IMAP4 implementation recommendations

http: //www . faqs . org/ftp/rfc/pdf/rfc2683 .txt.pdf

[33] D. Rogerson, Inside COM. Microsoft Press, USA, 1996. ISBN 1-572-31349-8.

[34] G. SALTON, Introduction to Modern Information Retrieval. McGraw-Hill, USA; 1983.

[35] G. Tambouratzis, C. CARAyannis, Automatic Corpora-based Stemming in Greek. Literacy and Linguistic Computing, 16 (2001).

[36] D. C. VEAL, Techniques of document management: A review of text retrieval and related technologies. Journal of Documentation, 57 (2001), pp. 192-217.

[37] T. A. WELCH, A technique for High Performance Data Compression. IEEE Computer, 17 (1984).
[38] AUTONOMY products:

http://www . autonomy.com/content/Products/ IDOL/

http://www . autonomy. com/content/Products/ IDOL_Desktop/

http://www . autonomy.com/content/Products/ Federator/

http://www . autonomy. com/content/Products/ Mobile/

http://www . autonomy.com/content/Products/ IDOL_K2/

[39] BONOBO

http://developer.gnome.org/arch /component/bonobo.html

http://ftp.cs.stanford.edu/cs/theory/pub /katiyar/misc/ole_vs_corba.html

[40] GOOGLE DESKTOP

http://desktop.google.com/about.html http://desktop.google.com/features.html http://desktop.google.com/screenshots .html

[41] HYPERION Technical Overview http://www.sirsidynix.com/Solutions /Products/digitalarchive.php http://www.sirsidynix.com/Resources /Pdfs/Solutions/Products/Hyperion _Technical_Overview.pdf

[42] IJG (Independent JPEG Group) free JPEG software ftp://ftp.uu.net/graphics/jpeg/jpegsrc .v6b.tar.gz ftp://ftp.simtel.net/pub/simtelnet /msdos/graphics/

[43] JAVAMAIL http://java.sun.com/products/javamail/

[44] LIBESMTP (C language SMTP) http://www.stafford.uklinux.net/libesmtp

[45] MAPI: Messaging (Mail) Application Programming Interface http://www. eudora.com/developers/mapi .html

[46] Motion JPEG 2000 (MJ2) http: //www.jpeg.org/jpeg2000/j2kpart3 .html

[47] MOZILLA THUNDERBIRD http://www.mozilla.org/products /thunderbird/

[48] MPEG Organization http://www.mpeg.org/ http://www .mpeg.org/MPEG/startingpoints.html

[49] NEXTPAGE

http://www. nextpage.com/products /features.htm http://www. nextpage.com/products /technology.htm

[50] PHPMAIL http: //www . phpmail .com

[51] PYTHON SMTP http://www.eskimo.com/ jet/python /examples/mail/smtp1.html 
[52] RTF Version 1.5. Rich Text Format (RTF) Specification and Sample RTF Reader Program. Application Note, Microsoft Technical Support, 1997. http://www.snake.net/software/RTF/ RTF-Spec-1.5.pdf http://www.snake.net/software/RTF/ RTF-Spec-1.5.rtf

[53] SANE

http://www.sane-project.org/intro.html http://www.sane-project.org/lj98 /doc000.html

[54] TIFF 6.0 specification. http://partners.adobe.com/public /developer/en/tiff/TIFF6.pdf ftp://ftp.faximum.com/pub/documents /TIFF6.pdf

[55] TWAIN Organization http://www.twain.org/

[56] TWAIN Version 1.6, February 5, 1996, Part Number 100-15008.

http://www . twain.org/docs/TWAIN-16Spec.pdf

[57] TWAIN v1.7, Vision Statement \& Functional Specification, Tuesday, September 16, 1997. http://www.twain.org/archive/spec17.doc

[58] Expanding TWAIN's portability to Include Unix http://www. twain.org/docs/twain_20_unix .htm

[59] VIDEO FOR LINUX

http://www. thedirks.org/v412

http://linux.bytesex.org/v412

http://www.video4linux.net/

[60] VIDEO FOR WINDOWS (VfW)

http://www. videoforwindows. com/samplecode/ http: //www.videoforwindows.com/articles/ http://ej.bantz.com/video

[61] XIMIAN EVOLUTION

http://www.gnome.org/projects/evolution
Received: October, 2005 Revised: April, 2006 Accepted: May, 2006

Contact address: Nikitas N. Karanikolas Technological Educational Institute of Athens (TEI-A)

Department of Informatics Ag. Spyridonos Street

Athens

Greece

e-mail: nnk@teiath.gr

DR. NIKITAS N. KARANIKOLAS received his Diploma in computer science from the Athens University of Economics and Business, Greece, in 1988 and his Ph.D. degree in 1994, from the same University. He has been a professional/practitioner in software development and support since 1989. In 1996, he joined Technological Educational Institute of Athens (TEI-A) as Systems' Head of Library. He worked as Systems' Head of Areteion University Hospital for a long period (from October 1997 until November 2004). Since November 18, 2004 he has been an Assistant Professor in the Department of Informatics of Technological Educational Institute of Athens. His current research interests lie in computational linguistics, natural language understanding, multilingual information retrieval, text data mining, information extraction, medical informatics, e-commerce and e-government. He is General Secretary of the Board of Directors of the Greek Computer Society. 